\title{
DISCURSOS GEOPOLÍTICOS DE DESARROLLO Y REESTRUCTURACIÓN TERRITORIAL IIRSA EN EL EJE MERCOSUR-CHILE*
}

\author{
GEOPOLITICAL DISCOURSES OF DEVELOPMENT AND TERRITORIAL \\ RESTRUCTURING IIRSA IN THE MERCOSUR-CHILE AXIS
}

\author{
Pablo Mansilla Quiñones ${ }^{* *}$, Alexander Panez Pinto ${ }^{* * *}$ y María Ignacia Ponce-Hille M**** $^{*}$
}

\begin{abstract}
La Iniciativa para la Integración de la Infraestructura Regional Suramericana (IIRSA) compone una de las estrategias geopolíticas de reestructuración territorial y económica más ambiciosas de América Latina. Sus impactos socioambientales han sido denunciados en diversos países de la región, sin embargo, para el caso de Chile, existe escasa evidencia empírica. En este artículo se analizan las consecuencias territoriales y ambientales para el caso del corredor Mercosur-Chile, analizando las estrategias discursivas de los promotores del IIRSA respecto de desarrollo, integración y territorio que proponen. Posteriormente se devela el modo en que se ensamblan inversiones económicas a este eje. Y se analizan los impactos socioambientales generados por el corredor bioceánico y la expansión del puerto de Valparaíso. Los resultados permiten evidenciar el modo en que los discursos de desarrollo ocultan los verdaderos impactos territoriales que suponen los proyectos.
\end{abstract}

Palabras claves: IIRSA, desarrollo, territorio, integración, infraestructura.

The Initiative for the Integration of Regional Infrastructure in South America-IIRSA is one of the most ambitious geopolitical strategies for territorial and economic restructuring in Latin America. Its socio-environmental impacts have been reported in several countries of the region, however, in the case of Chile, there is scarce empirical evidence. This article analyzes the territorial and environmental consequences for the case of the Mercosur-Chile corridor, analyzing the discursive strategies of the IIRSA promoters on: development, integration and territory that they propose. Subsequently, the way in which economic investments to this axis are assembled is revealed. And the socio-environmental impacts generated by the bioceanic corridor and the expansion of the port of Valparaíso are analyzed. The results show the way in which the discourses of development hide the true territorial impacts that the projects suppose.

Key words: IIRSA, geopolitics, development, territory, integration, infrastructure.

\section{Introducción}

El contexto político y económico actual nos enfrenta a un proceso de reestructuración geopolítica del sistema mundo moderno/colonial, que se expresa primero en la disputa por hegemonía desde Asia, y la pérdida de poder de Estados Unidos y Europa (Wallerstein, 2007). Al mismo tiempo, un cambio geopolítico interno en América Latina, en que Brasil se ha venido perfilando cada vez más como una posible potencia sudamericana, materializado en ciertas políticas subimperialistas respecto de los países vecinos fundamentadas por el extractivismo y la acumulación por desposesión ${ }^{1}$ de bienes comunes naturales (Zibechi, 2012).
Estos cambios en las correlaciones de fuerza han tenido como consecuencia un significativo proceso de desterritorialización y reestructuración territorial, siendo uno de los más evidentes la Iniciativa de Integración Regional de América del Sur (IIRSA) (Dijck, 2013). Durante la última década, esta iniciativa ha venido promoviendo a escala continental la integración económica regional mediante el despliegue de una alianza estratégica de megainfraestructuras -como vías, puertos, aeropuertos, entre otros- que faciliten la movilidad de materias primas (Madrid et al., 2011). Como lo señala Zibechi (2012), por medio de este despliegue técnico se busca vencer las barreras físicas, legales y sociales para el capital, lo que

\footnotetext{
* Resultado Proyecto Conicyt-PIA Anillos SOC180040.

** Pontificia Universidad Católica de Valparaíso, Chile. Correo electrónico: pablo.mansilla@pucv.cl

*** Universidad Nacional Andrés Bello, Viña del Mar, Chile. Correo electrónico: alexander.panez@gmail.com

**** Pontificia Universidad Católica de Valparaíso. Instituto de Geografía. Laboratorio Geografía Social y Territorialidades Alternativas, Chile. Correo electrónico: poncehille.gonzalez@gmail.com
} 
supone rediseñar la geografía, la legislación de los Estados y las relaciones sociales (Zibechi, 2012). El proyecto IIRSA pretende facilitar la exportación de commodities hacia las hegemonías político-económicas del Asia-Pacífico, en particular China. Originalmente, posicionando a Brasil como corazón geopolítico de los flujos provenientes desde América Latina (Zibechi, 2012). Los proyectos IIRSA tienen una significativa vinculación con la trayectoria de la geopolítica brasileña, en cuanto legado de la visión de desarrollo reproducida en la dictadura militar, que promueve la Constitución de Brasil en el heartland político del continente. En efecto, es posible evidenciar una continuidad en los planteamientos geopolíticos de los militares brasileños, como es el caso de Golbery do Couto e Silva, quien proponía promover la influencia imperial de Brasil en América Latina de manera pacífica mediante la consolidación de un proyecto nacional que posteriormente facilitará un posicionamiento geopolítico en el contexto sudamericano promoviendo su visión de desarrollo. Para esto era necesaria la alianza con Estados Unidos, y la concreción de acciones geopolíticas como la articulación geográfica interior, integrando y expandiéndose en el territorio nacional y con un control sobre espacios fronterizos (Gallardo et al. 2012). En este proyecto las megainfraestructuras de transporte juegan un rol fundamental.

Los proyectos IIRSA han sido cuestionados durante la última década por diversos intelectuales latinoamericanos y por los propios movimientos sociales afectados (Pieck, 2011; Porto-Gonçalves y Quental, 2012: Zibechi, 2012; Quintero-Weir, 2015). Estos evidencian entre las consecuencias más significativas de la IIRSA, su impacto en la Amazonia, motivando severos conflictos territoriales entre pueblos originarios, movimientos medioambientales y promotores públicos y privados de los proyectos de infraestructura (Pieck, 2011).

No obstante, es posible evidenciar que en Chile no se ha generado una discusión en profundidad al respecto, lo que es notoriamente contradictorio considerando que el territorio nacional es atravesado por diversos ejes.

Uno de los más relevantes en términos de población comprometida, volumen de producción y dinero es el eje Mercosur-Chile, integrado por Brasil, Argentina, Paraguay, Uruguay y Chile. Analizar los impactos de este eje resulta relevante en la medida en que se reconoce que los países involucrados han experimentado un profundo proceso de reestructuración neoliberal durante las últimas décadas, lo que ha tenido como consecuencia un retroceso del Estado en la planificación territorial, traspasando dichas funciones al mercado. Este eje presenta a Brasil como potencia y motor de "desarrollo", Argentina como productor de commodities asociados a la agroindustria y Chile con potencial logístico para la salida de los productos hacia el Asia-Pacífico. Si bien el eje atraviesa territorio uruguayo y paraguayo, estos países no son mencionados dentro los discursos de desarrollo asociados al eje Mercosur-Chile, cumpliendo un rol secundario que no se encuentra bien definido. De esta forma, el objetivo del presente artículo es comprender las consecuencias territoriales y ambientales generadas por el corredor Mercosur-Chile, mediante el análisis de la relación entre los discursos de desarrollo geopolítico y las transformaciones territoriales y socioambientales que se desprenden de los proyectos de este eje.

Para ello se analizan los impactos territoriales en tres dimensiones: a) la reestructuración geopolítica que supone la consolidación del eje Mercosur-Chile, b) los impactos ambientales que se vislumbran en la región de Valparaíso, y c) los discursos pertinentes al desarrollo, la integración y el territorio en los que se sustentan los proyectos. Posteriormente se analizan los impactos territoriales y socioambientales a partir de herramientas de sistemas de información geográfica.

Los resultados permiten evidenciar la desconexión entre el pretendido desarrollo territorial propuesto por el eje, debido a su contraste con los impactos socioambientales realmente generados.

\section{Metodología}

El abordaje metodológico es de tipo mixto. En la primera parte del artículo el análisis cualitativo se generó a partir de la revisión de fuentes secundarias de documentos institucionales, instrumentos de planificación territorial y discursos de autoridades que promueven el plan IIRSA, utilizando herramientas de análisis crítico de los discursos de desarrollos que acompañan a los proyectos IIRSA. Las categorías de análisis discursivo utilizadas fueron: i) Desarrollo, ii) Integración y iii) Territorio.

Posteriormente se realiza una revisión cuantitativa de carácter descriptivo para dar cuenta del contexto territorial en el que se inscribe el 
eje IIRSA Merco-Sur Chile. El análisis espacial de los indicadores se realizó a base del cálculo de densidad espacial Kernel de proyectos de inversión aprobados en el Sistema de Evaluación de Impacto Ambiental (SEIA), en el periodo 20002018, localizados en un área de influencia de 10 kilómetros respecto de los proyectos IIRSA en la Región de Valparaíso, otorgando pesos con mayor valor a los proyectos de tipo portuario, transportes y energéticos. Para el análisis de los conflictos ambientales y territoriales se generó análisis espacial vectorial por superposición de polígonos acerca de información espacial de Áreas Protegidas en la Región de Valparaíso localizadas en un área de 10 kilómetros de los proyectos IIRSA. El resultado de la intersección de estas informaciones permitió estimar el área intervenida. Se identifican también dos conflictos en particular, geolocalizados a partir de los sectores con mayor conflictividad con las localidades intervenidas directamente.

\section{Geopolítica sudamericana y el surgimiento de la IIRSA}

Brasil ha jugado históricamente un rol preponderante dentro de la geopolítica sudamericana en las relaciones de integración-dependencia. En este sentido, un hito de las aspiraciones de protagonismo de las élites brasileñas dentro del escenario regional fue el gobierno de Getulio Vargas, que a mediados del siglo XX buscó consolidar a Brasil como una potencia regional, a partir del desarrollo de la economía interna, impulsando la industrialización y la llamada conquista del Oeste brasileño. Sin embargo, el posicionamiento geopolítico se consolida luego del golpe de Estado de 1964, en el que se develan con más claridad las pretensiones subimperialistas (Marini, 1977). Es importante destacar lo que representa este golpe de Estado para la historia de América Latina, según Marini (1977) y Zibechi (2012), no se trató de una mera interferencia de Estados Unidos que utilizó como "títeres" a los militares y a la burguesía brasileña. Al mismo tiempo que la intervención militar tampoco puede ser leída como una acción independiente por parte de las clases dominantes en Brasil. La intervención de Estados Unidos fue significativa, como lo demuestran las acciones previas de apoyo al golpe coordinadas por su embajador en Brasil, Lincoln Gordon ${ }^{2}$. Sin embargo, en ese proceso también se puso en juego la aspiración de consolidar un proyecto político nacional detrás del golpe, defendido por los militares, como lo evidencian las inversiones en el complejo industrial-militar (Marini, 1977) y, particularmente, el interés por el desarrollo de energía nuclear en Brasil. De esta forma, la dictadura militar se propuso la meta de conciliar la política de hegemonía de Estados Unidos con las aspiraciones de la burguesía nacional brasileña. Esta alianza entre la burguesía nacional con los altos mandos militares promovió un proceso de modernización conservadora, con el objetivo de acelerar el crecimiento económico mediante la industrialización del país ${ }^{3}$.

El crecimiento económico supuso la necesidad de que los capitales nacionales se expandieran fuera de las fronteras del Brasil, promoviendo la acumulación por desposesión, valiéndose incluso de medios de fuerza mediante estrategias de control militar de los territorios de expansión económica, lo que se plasma en los planes de invasión en Uruguay en 1970, el apoyo al golpe de Estado en Bolivia en 1971 y las agresiones militares en Paraguay que forzaron la construcción de la represa Itaipú en 1973 (Zibechi, 2012). Basados en esta trayectoria se explica que el origen de la iniciativa IIRSA haya sido intensamente promovida por Brasil, como continuidad histórica de su posición geopolítica, en la que se refleja la influencia de pensadores como el barón de Rio Branco, quien marcó desde el siglo XIX el diseño de la política y diplomacia, influyendo el proyecto territorial brasileño hasta el tiempo presente.

La incorporación del modelo neoliberal, durante el periodo presidencial de Fernando Henrique Cardoso, también demuestra la continuidad de la influencia geopolítica de Brasil en América Latina. Por medio de acuerdos políticos que promovió durante su gobierno orientados a la apertura regional, mediante el desarrollo de megainfraestructuras de transporte que permitieran consolidar una red logística a escala continental para el transporte de materias primas (Quintanar y López, 2003). Es importante señalar que esta oleada neoliberal de integración se da bajo la perspectiva del regionalismo abierto, o nuevo regionalismo, que sería

una forma de pensar América del Sur como un espacio geoeconómico integrado, siendo necesaria la reducción de barreras internas al comercio, apertura 
en la infraestructura y en los sistemas de regulación y de operación que apoyan las actividades de producción en escala regional (Porto-Gonçalves, 2011).

Este movimiento regional liderado por Brasil no implicó que Estados Unidos adquiera un rol pasivo en su función de principal potencia en la región, por el contrario, tuvo un rol protagónico en el surgimiento de la IIRSA, a través del Banco Interamericano de Desarrollo (BID) que prestó financiamiento y asesoría técnica en los proyectos IIRSA desde su inicio. Para Estados Unidos, la IIRSA representaba el soporte de infraestructuras para implantar el ALCA (Área de Libre Comercio de las Américas) en la región.

Según Porto-Gonçalves y Quental (2012), el IIRSA encuentra también sus orígenes en las políticas establecidas desde la década de 1990, mediante la política de los Ejes Nacionales de Integración y Desarrollo (ENID), cuyos objetivos eran establecer una red logística que permitiera promover la competitividad de los productos de Brasil, incorporar nuevas áreas del país al comercio global, y la consolidación de la hegemonía política y económica de Brasil en América del Sur ${ }^{4}$.

El proyecto geopolítico brasileño históricamente ha comprendido la necesidad de conseguir una salida hacia el océano Pacífico para consolidar su poder. El cambio de la hegemonía hacia la cuenca del Pacífico genera que esto se transforme en una necesidad concreta, así como lo señala Zibechi

\section{El tráfico comercial y portuario del Pacífico desplazó al del Atlántico lo que supone reinventar la economía de un Océano al otro. Eso ha hecho que el canal de Panamá pierda su importancia y en su lugar apare- cen estos corredores para conectar ambos océanos (2012: 207).}

En esta participación subimperial de Brasil no podemos perder de vista que la región sudamericana se encuentra en una condición de "doble subordinación". Por un lado, la hegemonía de Brasil sobre el resto de los países de América del Sur, que busca priorizar los intereses de sus capitales en la actividad económica de la región. Y al mismo tiempo, la subordinación del subcontinente a las tensiones territoriales de la tríada Estados Unidos-Europa Occidental-Asia Oriental (Wallerstein, 2007), que disputan y negocian la expansión de la acumulación capitalista.

Es importante señalar que dicha integración no ha tenido oposición por parte del resto de los gobiernos de la región, ya que incluso gobiernos progresistas de izquierda como Ecuador, Venezuela o Bolivia, han impulsado la cartera de proyectos asociados a la IIRSA, generando notorias contradicciones y conflictos territoriales con los movimientos sociales (Pieck, 2011). Una muestra de esto es la importancia que adquiere UNASUR como coordinadora de la iniciativa por medio del Consejo Suramericano de Infraestructura y Planeamiento (COSIPLAN), a partir del 2009.

Como ha sido documentado por otros autores (Svampa y Slipak, 2015; Moreno, 2015), Sudamérica ha vivenciado un incremento explosivo de las relaciones comerciales con $\mathrm{China}^{5}$, que han consolidado a este país como el principal socio comercial de la región, lo que se expresa en los volúmenes de exportación de materias primas y la creciente inserción de empresas con capitales chinos. Estados Unidos, frente al fracaso parcial de sus iniciativas de articulación económica en el continente (ejemplo, ALCA), intenta retomar su protagonismo en América Latina. Como es tradicional en su política exterior, ha apoyado económica y políticamente a sectores más proclives a sus intereses para cambiar la correlación de fuerzas en los gobiernos sudamericanos, como es el caso de Argentina, Brasil, Venezuela y Ecuador.

Un elemento importante en la dinámica contemporánea en la gestión de IIRSA-COSIPLAN es el reacomodo de fuerzas geopolíticas en la implementación de los proyectos de infraestructura, que se comienza a apreciar con mayor notoriedad a partir del 2014. Las denuncias de corrupción en Brasil, surgidas a partir de la investigación LavaJato, junto con la disminución del financiamiento de proyectos de infraestructura fuera de Brasil por parte del BNDES (Banco Nacional de Desarrollo Económico y Social) debido a la crisis fiscal en ese país, incidió en la disminución del protagonismo del gobierno brasilero y de las empresas de consultoría y construcción civil de ese país en la implementación de la IIRSA, dejando espacio para la inserción de otros actores que hasta ese momento no tenían un protagonismo directo en dicho plan. Particularmente, la nueva política china de ampliar sus inversiones en América del Sur hacia el financiamiento directo y la ejecución de infraestructuras para incrementar la circulación de 
mercancías, es un elemento que está marcando una nueva tendencia en la ejecución de los proyectos de IIRSA-COSIPLAN (Aguiar, 2017).

\section{Eje Mercosur-Chile}

Es en este escenario de tensiones en torno a la doble subordinación de América del Sur que el eje MCC aparece como uno de los puntos claves de la estrategia IIRSA. Como muestra en la Figura 1, este corredor corresponde al $18 \%$ de la superficie de Sudamérica, extendiéndose de norte a sur desde el estado de Minas Gerais y comprende la costa sudeste de Brasil, Uruguay y el litoral de Argentina hasta la provincia de Buenos Aires. En dirección hacia el Oeste incluye a Paraguay, la zona central de Argentina y Chile hasta el Pacífico.

Desde el punto de vista demográfico, su relevancia es clave, ya que cubre el $34,9 \%$ de la población de América del Sur, siendo el eje con mayor población de la IIRSA (Valdés y Parimbelli, 2014). En lo referido a la actividad económica, el Producto Interno Bruto (PIB) de las unidades administrativas de los países que forman el eje equivalen al 47,7\% del PIB de Sudamérica (primer lugar del conjunto de ejes de IIRSA). Del total de ese volumen de producción, Brasil participa con el $67 \%$ (Valdés y Parimbelli, 2014).

Según la información entregada por el Sistema de Información de Proyectos IIRSA ${ }^{6}$, el Eje Mercosur-Chile cuenta con una inversión total de US\$ 58.515.621,2 millones destinados a 119 proyectos en tres ámbitos sectoriales: transporte, energía y comunicaciones, lo que supone $25 \%$ del total de los nueve ejes multimodales que conectan los océanos Atlántico y Pacífico. Como se evidencia en la Figura 1, Argentina es el país con mayor aporte financiero al Eje Mercosur-Chile, con US\$ 33.978 millones que se distribuyen en 49 proyectos, de manera equitativa entre energía $\mathrm{y}$ transporte, $\mathrm{y}$ tan solo 8 corresponden al sector energético, mientras que los 41 proyectos restantes son de transportes, es decir, las obras energéticas son de mayor magnitud y, por esta razón, necesitan de un alto gasto financiero, no existiendo relación directa en Argentina entre el financiamiento y el número de proyectos por sector.

Brasil por otra parte se encuentra en el segundo lugar de inversión, destinando US\$ 16.630 millones en 20 proyectos de transportes. Y US\$ 3.736 millones para el desarrollo de 5 proyectos energéticos.
Chile y Paraguay muestran un comportamiento completamente opuesto. Por una parte, Chile concentra proyectos de un solo sector, en este caso transporte, cuyo presupuesto de $\$ 7.921$ millones de dólares corresponde a 19 proyectos. Muy por el contrario, el $97 \%$ de la inversión total correspondiente a 9.465 millones de dólares se encuentra destinado a 4 proyectos del sector energético, mientras que el $3 \%$ restante corresponde a proyectos menores de transporte. Finalmente, Uruguay es el país con menor aporte al Eje Mercosur-Chile, sin embargo, con la inversión total de 3.161 millones de dólares permite la ejecución de 29 proyectos en su territorio, 20 de ellos destinados al sector de transportes financiados por 1.970 millones de dólares, solo 8 proyectos energéticos a 1.190 millones de dólares a su favor $\mathrm{y}$ un proyecto comunicacional que al igual que en Brasil no destina financiamiento para ese sector.

En lo que respecta a proyectos binacionales, se puede señalar que estos corresponden al 13\% de los proyectos, así como se señala en el mapa, se observa que las relaciones binacionales se concentran solo en el sector energético y transporte. En el primer caso, se muestra como predominante, destacando que la mayor relación internacional con una cantidad de 5 proyectos es Brasil-Uruguay, seguido por ArgentinaParaguay y Brasil-Paraguay, es este último quien llama más la atención, ya que según financiamiento posee la mayor inversión de los proyectos binacionales, son 16.000 millones de dólares destinados para el Sistema de Itaipú, la mayor central productora de energía eléctrica del mundo en la actualidad, lo que significa que Argentina y Brasil son quienes poseen una fuerte influencia geopolítica en el Eje Mercosur-Chile. Por otra parte, se observa que solo existen relaciones internacionales vinculadas al transporte entre Chile y Argentina, con cinco proyectos binacionales ocupan el primer lugar en proyectos binacionales del eje Mercosur-Chile, el financiamiento total es de 3.230 millones de dólares, de este modo se podría apuntar a que Chile cumple un rol logístico.

Dentro de la dinámica comercial del eje, Chile con sus 4.329 km de costa Pacífico en el territorio continental, es un aliado clave para la integración económica de la estrategia IIRSA. En cuanto a las iniciativas IIRSA en el país, la región de Valparaíso se destaca por proyectarse la implementación de 9 proyectos, que concentran el $45,6 \%$ de recursos de la cartera total en el país, que asciende a aproximadamente a 16.105 millones de dólares, como se puede apreciar en la Tabla 1. 


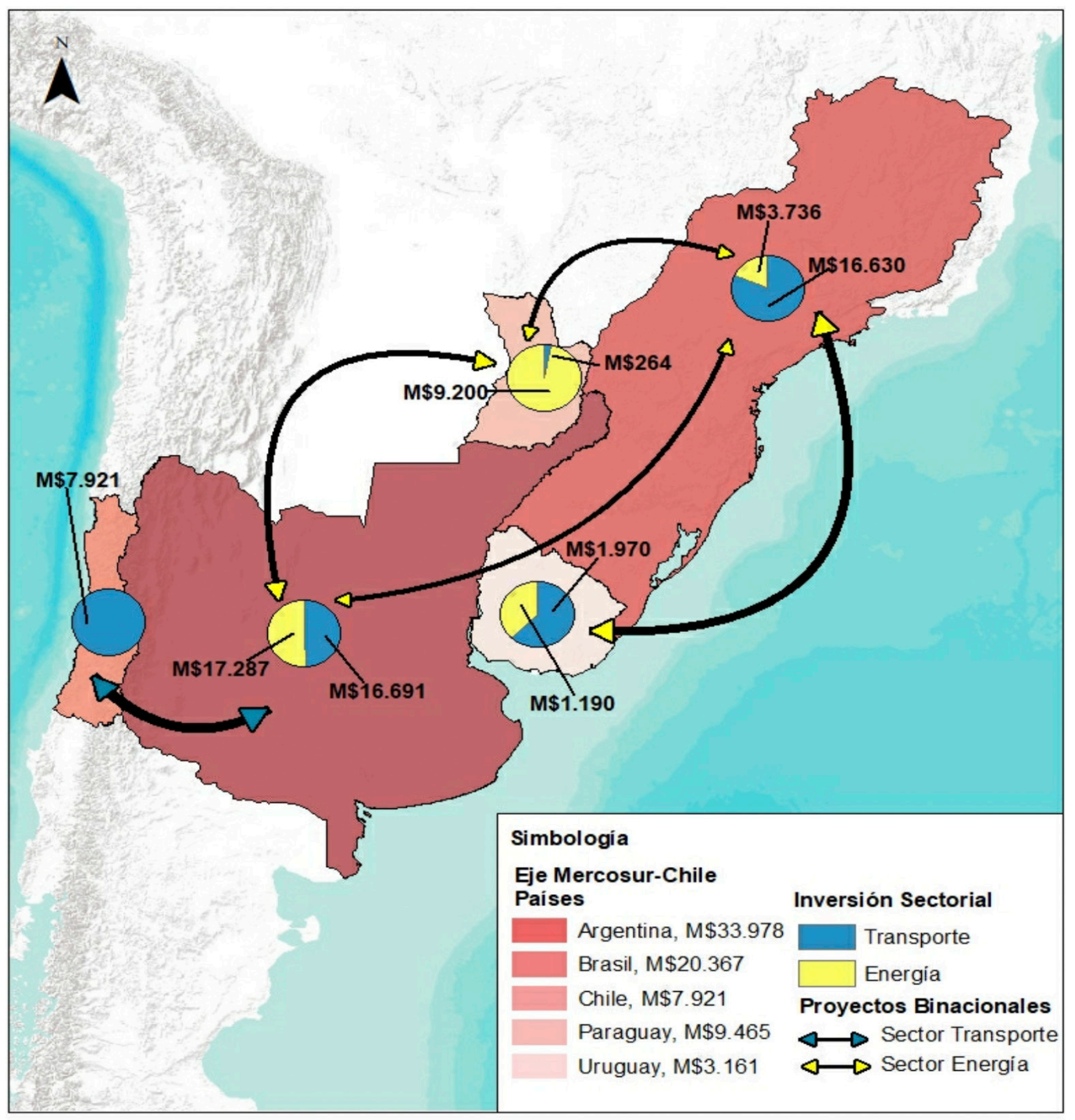

Figura 1. Financiamiento por país en el eje Mercosur-Chile.

Fuente: Elaboración propia basada en datos oficiales de IIRSA (2018).

Tabla 1. Proyectos IIRSA en la región de Valparaíso.

\begin{tabular}{lll}
\hline \multicolumn{1}{c}{ Nombre del proyecto } & Etapa proyecto & \multicolumn{1}{c}{ Inversión (en US\$) } \\
\hline Mejoras al acceso vial puerto de Valparaíso & Concluido & 105.000 .000 \\
Puerto terrestre Los Sauces (Los Andes) & Concluido & 61.000 .000 \\
Ruta Internacional CH-60 (sector Valparaíso-Los Andes) & Ejecución & 447.000 .000 \\
Mejoras en el puerto de San Antonio & Ejecución & 370.000 .000 \\
Ampliación del puerto Valparaíso & Ejecución & 560.000 .000 \\
Nuevo complejo fronterizo Los Libertadores & Ejecución & 76.000 .000 \\
Ruta de la fruta: San Antonio-San Fernando & Pre-ejecución & 600.000 .000 \\
Readecuación túnel Cristo Redentor y Caracoles & Pre-ejecución & 770.000 .000 \\
Sistema binacional de control de gestión del paso Cristo Redentor & Pre-ejecución & Sin valor en la página oficial \\
\hline
\end{tabular}

Fuente: Elaboración propia. 
Estrategias discursivas de los proyectos IIRSA en la región de Valparaíso

\section{a) Sobre el desarrollo}

Los instrumentos de planificación regional de Valparaíso componen una retórica acerca del desarrollo sostenible como meta de los proyectos propuestos asociados a este eje. No obstante, al revisar los proyectos impulsados se aprecia una mirada de desarrollo restringida únicamente a lo productivo. Muestra de esto es que el primer desafío estratégico definido (la estrategia regional de desarrollo de Valparaíso 2020) sea consolidar el proyecto del corredor bioceánico (Gobierno Regional de Valparaíso, 2012).

En los instrumentos de planificación de periodos anteriores ya se visualiza la relevancia que las autoridades regionales le otorgan a este proyecto IIRSA. Por ejemplo, la Estrategia Regional Desarrollo 2001-2010 define como objetivo en el ámbito de infraestructura:

\section{Fortalecer la modernización y expansión de la infraestructura marítima, terrestre, aérea y de comunicaciones, de los ser- vicios relacionados y de la red regional vial estructurante que otorgue viabilidad al corredor bioceánico o corredor de co- mercio ampliado del Cono Sur (Gobierno Regional de Valparaíso, 2001: 4).}

Las justificaciones de dichos proyectos intentan validarse por medio de una argumentación histórica tomando como referencia los modelos de redes de infraestructura que el ser humano ha desarrollado durante la historia de la modernidad, mostrando como principal ejemplo las redes desplegadas en Europa, y cómo estas permitirían avanzar en la búsqueda de posibilidades de desarrollo. Los países que más se han integrado físicamente han experimentado un aumento considerable de las condiciones de desarrollo económico y por consiguiente de la calidad de vida de sus habitantes. De esta forma, la implementación de estos proyectos va acompañada de un discurso concerniente al desarrollo, sustentado en los mitos de la modernidad/colonialidad (Escobar, 2007). Uno de estos mitos es la idea de "progreso de la humanidad" bajo la que se justifica el proceso de modernización en los países. Detrás de la promesa de progreso, diferentes proyectos modernizadores han demostrado ser desiguales en sus beneficios y excluyentes en sus impactos. Esto ocurre en la medida en que dichos proyectos privilegian una construcción hegemónica de lo que sería lo "civilizado/ moderno" y frecuentemente generan "sacrificados por el progreso", grupos subalternizados y territorios que son intervenidos y precarizados para lograr el bienestar de un determinado "centro".

En el caso particular de la región de Valparaíso, a pesar de las declaraciones de intenciones de mejorar las condiciones de vida de la población, los proyectos emblemáticos en la región no están dirigidos directamente a sus habitantes, sino que tienen como objetivo la intensificación de la circulación de mercancías por este territorio, como se demuestra más adelante en el análisis de sus proyectos emblemáticos.

\section{b) Sobre la integración}

Como ha sido documentado en buena parte de la literatura crítica de la IIRSA (Porto-Gonçalves y Quental, 2012; Zibechi, 2015), el concepto de integración que está contenido en la iniciativa es profundamente restringido, remitiéndose principalmente a aspectos económicos. Esta forma de comprender la integración también se evidencia en los discursos de las instituciones vinculadas a los proyectos del eje MCC en la región de Valparaíso.

En el caso del Ministerio de Obras Públicas, en su Plan Regional de Infraestructura y Gestión hídrica, explicita la prioridad de sus esfuerzos:

\section{Finalmente, respecto de la competitividad económica-logística, las siguientes iniciati- vas consolidarán a futuro a la región como el principal polo logístico nacional, y uno de los más importantes del Cono Sur: el túnel de baja altura Los Andes-Mendoza; la expansión portuaria de Valparaíso y San Antonio, y su rol como principal sistema portuario de la Costa Oeste sudamericana" (2012: 121).}

Por otra parte, es llamativo que, dentro de las estrategias discursivas en torno a la idea de integración, sea utilizada una retórica latinoamericanista, como el ejemplo ya mencionado del proyecto del Corredor Bioceánico, en donde se hace alusión a la unidad entre los "libertadores de la patria" de Chile y Argentina para justificar dicha iniciativa. 
Es constante la referencia a la necesidad de estos proyectos, apelando a que se trata de países hermanos que requieren estar más integrados. No obstante, ambos proyectos emblemáticos no están destinados a la mejora del flujo de personas o de la comunicación entre los países involucrados, lo que daría cuenta de una integración más sustantiva.

Ahora bien, otro aspecto vinculado al discurso de la integración tiene que ver con las estrategias de inserción de estos proyectos mediante los discursos institucionales. Llama la atención que en la mayoría de los instrumentos de planificación revisados en Chile (elaborados por el gobierno regional, el Ministerio de Obras Públicas y el Ministerio de Relaciones Exteriores) y en los discursos de autoridades, la referencia a IIRSA, COSIPLAN y UNASUR es casi inexistente. Esto tiene que ver con el modo de inserción que ha tenido esta iniciativa, que invisibiliza la relación de cada uno de los proyectos propuestos con la estrategia de reestructuración territorial que subyace en el plan IIRSA. Como señala Zibechi:

\section{la forma como se está implementando [el plan IIRSA] es muy grave, ya que los proyectos se están llevando adelante en silencio. Así como en todo el continente hay un amplio debate sobre el ALCA y los $T L C$, los proyectos vinculados a la IIRSA se vienen realizando sin participación de las sociedades civiles ni de los movimientos sociales, sin información por parte de los gobiernos (2006: 6).}

No obstante, en su trayectoria de implementación, y debido principalmente a las acciones de movimientos y comunidades, en algunos países (como Bolivia, Ecuador o Brasil) comenzó a abrirse en la última década una discusión pública por los alcances de los proyectos IIRSA y sus supuestos respecto de la integración sudamericana. Esta apertura al debate y conocimiento público de la IIRSA no ha ocurrido en el caso chileno. La persistencia en el silencio representa una particularidad de la implementación de la IIRSA en Chile, como lo señala también el trabajo de Jiménez (2014). Independiente de que sea o no un objetivo pretendido por las autoridades chilenas, la consecuencia de esta política de silencio es la neutralización de posibles conflictos sociales. Si para la existencia de un conflicto se requiere como elemento básico la divergencia entre, por lo menos, dos posiciones diferentes, la poca transparencia respecto de la relación que cada proyecto tiene con la estrategia mayor de reestructuración territorial que involucra IIRSA, imposibilita el posicionamiento del resto de los actores que son afectados por esta iniciativa.

\section{c) Sobre el territorio}

\section{La cordillera de los Andes es ciertamente una belleza, pero es un terrible problema de ingeniería (Carlos Lessa, expresidente del BNDES de Brasil, 2003).}

A diferencia de los mitos coloniales por el "vacío demográfico" ("territorios sin gente" que requieren ser conquistados) que acompañan a la implementación de la IIRSA en otros territorios de América del Sur (Porto-Gonçalves y Quental, 2012; Ceceña, 2008), por las características que posee el eje de "integración y desarrollo" MercosurChile de concentración de la mayor población de Sudamérica, altamente urbanizado, son otros los mitos que se ponen en juego para fabricar consensos y evitar oposiciones.

La mayoría de los territorios comprendidos en este eje, forman parte de lo que los planificadores de la IIRSA denominan "espacios consolidados" (Valdés y Parimbelli, 2014). A partir de la revisión de documentos institucionales y en los discursos de las autoridades, aparecen dos elementos centrales en los que se sustentan los proyectos del eje MCC en la región de Valparaíso; a) la cordillera como barrera natural para el desarrollo económico, b) el borde costero como oportunidad para la integración productiva de la región con el Pacífico sur.

En lo referido a la cordillera de los Andes como barrera natural, es reiterativa esta caracterización en los diferentes niveles de gestión y ejecución del plan IIRSA. Van desde los gerentes y especialistas de las agencias sudamericanas involucradas (CAF, UNASUR, entre otras), pasando por las autoridades nacionales de los países hasta llegar a los políticos y tecnócratas locales. En el caso chileno, un discurso que sintetiza esta visión de la cordillera es el de José Ortega Julio, coordinador de asuntos internacionales de la Dirección de Vialidad, que cuando se refiere a los corredores bioceánicos sostiene:

es uno de los grandes desafíos para enfrentar esta gran barrera económica que 
es la cordillera de los Andes. Saltando esta gran barrera económica, nuestros países van a poder hacer una cantidad de negocios muy importante, desarrollar un programa logístico que sirva a toda la región (COSIPLAN, 2016).

De esta forma es posible apreciar la capacidad de penetración de estos discursos moderno/coloniales, logrando el alineamiento de los discursos de autoridades y planificadores a nivel sudamericano, nacional y local.

En esta idea de la cordillera como barrera natural subyace un viejo discurso que acompañó la historia de la colonización de América Latina. Los conquistadores no solo tenían que dominar a los habitantes originarios del continente, sino que también a los diversos biomas/ecosistemas que impedían su proceso de expansión. Esta dominación no solo es física sino también simbólica mediante discursos/dispositivos que subordinan e inferiorizan para justificar la explotación de los bienes comunes naturales. Como señala Alimonda,

\section{en el caso americano, la naturaleza fue subvalorada a tal punto que los coloniza- dores destruyeron su valiosa biodiversidad, para implantar monocultivos de especies exóticas para exportación (2011: 47).}

En el caso de la profunda intervención que se proyecta en la cordillera, esto se vincula con los avances de la técnica para crear objetos que transformen los territorios (Panez, Mansilla \& Moreira, 2018). Se argumenta que a medida que avanza la técnica gracias al conocimiento científico, los seres humanos somos capaces de poder resolver esos grandes problemas de ingeniería de los que hablaba Carlos Lessa en la cita con la que comenzamos este apartado. Es posible la "conquista" de espacios que la ingeniería no conseguía intervenir, lo que a su vez requiere ir acompañado de viejos/nuevos discursos acerca de la naturaleza que pretende ser "dominada". De este modo, se enaltece a la técnica y sus posibilidades, sin explicitar la relación que la técnica posee con los intereses de los actores que se apropian de ella (Porto-Gonçalves, 2006).

Otra consecuencia del plan IIRSA en la comprensión de los territorios son sus implicancias como estrategia de reestructuración territorial para el pensamiento geográfico respecto de América del
Sur y cada uno de los países, así como las formas de planificación y ordenamiento territorial. El plan IIRSA fortalece el pensar Sudamérica por medio de ejes, más que pensar países, regiones, biomas, etc. Estos ejes tienen su expresión espacial en corredores, la mayoría de ellos con una orientación horizontal desde el Atlántico hacia el Pacífico. Así, se abandona la lógica zonal que impulsaban ideas como el desarrollo regional, para concentrarse en la conexión entre puntos que son estratégicos en una lógica reticular de organización espacial para el llamado "crecimiento económico", sustentado en la política extractiva de explotación de commodities.

\section{Inversiones e impactos socioambientales del eje Mercosur-Chile}

En el eje mercosur-Chile, la región de Valparaíso adquiere valor para los planificadores debido a sus características logísticas que presenta su emplazamiento geográfico y la infraestructura vial y portuaria con la que cuenta, que la conecta con la cuenca del Pacífico, movilizando casi el 50\% de los contenedores de carga marítima a escala nacional (Ministerio de Obras Públicas, 2012).

Dichas características promueven la ejecución de una gran cantidad de proyectos a nivel regional, que si bien no forman parte oficial de la cartera de proyectos de la IIRSA, se vinculan con dicho plan. Al hacer un análisis en relación con el IIRSA, se obtiene que, desde los orígenes de la iniciativa hasta marzo de 2017, se han aprobado mediante el Sistema de Evaluación de Impacto Ambiental 160 proyectos comprendidos en un área de influencia de 10 kilómetros respecto de la ruta internacional 60-CH y el proyecto San Antonio-San Fernando, ambas infraestructuras viales propuestas por IIRSA en la Región de Valparaíso. En esta acotada área de análisis se encuentran proyectos clasificados por el Servicio de Evaluación Ambiental según las siguientes tipologías: energía, equipamiento, fabril, minería, portuaria, saneamiento y transporte, a los que por motivo de la presente investigación se les otorgó un peso determinado para establecer una relación con los sectores trabajados por IIRSA, entregando mayor valor a transporte, energía y portuarios.

Como se expone en la Figura 2, existen tres sectores con alta densidad de proyectos, que se encuentran vinculados con las rutas internacional 60-CH y el proyecto vial San Antonio-San Fernando. El primero de ellos en la zona industrial 


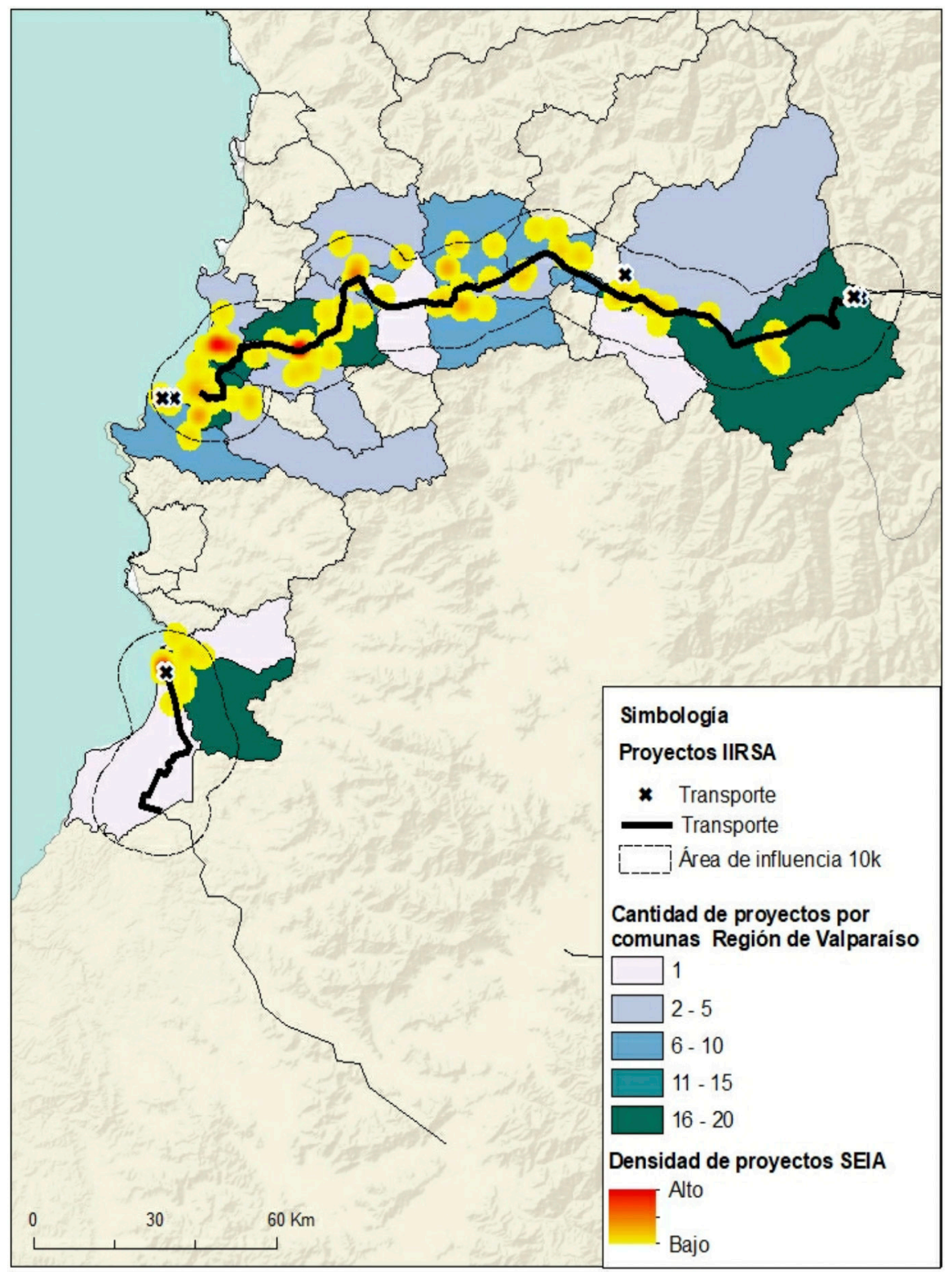

Figura 2. Densidad de proyectos SEIA aprobados en relación al IIRSA, Región de Valparaíso. Fuente: Elaboración propia en base datos oficiales de IIRSA.

de Concón, a un costado de la desembocadura del río Aconcagua, área destacada por contener una gran cantidad de industrias en un acotado territorio, en su mayoría de tipo energéticos, que al momento de realizar sus trabajos son usuarios constantes de la ruta $\mathrm{CH}-64$, ya que por ella realizan la conectividad con la ruta internacional $\mathrm{CH}-60$. El segundo caso se encuentra en la comuna de San Antonio, la que se ha transformado en un territorio atractivo para las inversiones del sector portuario debido a las mejoras en el Puerto y su conectividad con la VI Región, que propone la IIRSA. Finalmente, la tercera alta densidad identificada se encuentra entre las comunas de Quillota y Limache, en este sector la mayor cantidad de proyectos es de tipo energético relacionadas con las termoeléctricas San Isidro y Nehuenco, más el nuevo proyecto energético Los Rulos, transformando esta área 
Tabla 2. Proyectos aprobados por SEIA que se encuentran localizados a $10 \mathrm{~km}$ de distancia del Eje Mercosur Chile.

\begin{tabular}{lc}
\hline \multicolumn{1}{c}{ Tipo de proyecto } & Cantidad de proyectos \\
\hline Energía & 51 \\
Equipamiento & 35 \\
Fabril & 20 \\
Minería & 21 \\
Portuaria & 11 \\
Saneamiento & 11 \\
Transporte & 11 \\
\hline
\end{tabular}

Fuente: Elaboración propia basado en el Sistema de Evaluación de Impacto Ambiental SEIA, abril del 2019.

en una de las zonas que se encuentra en mayor conflicto durante el último tiempo. De este modo se puede demostrar que la IIRSA al trazar sus redes otorgan un valor agregado al territorio local, generando una importante atracción de proyectos e inversiones.

Por otra parte, existen 21 comunas que son intervenidas por el área de influencia definido a partir del IIRSA, permitiendo la comparación gráfica de aquellas que poseen entre 16 y 20 proyectos en sus territorios, identificando Concón, San Antonio, Quillota, Viña del Mar y Los Andes. Las tres primeras comunas presentan correlación con el análisis pertinente a densidad, mientras que Los Andes y Viña del Mar adquieren importancia por ser el inicio y fin de la ruta internacional $\mathrm{CH}-60$, en el primer caso los proyectos son de carácter energético presentados por la División Andina de la empresa CODELCO, sumando los proyectos de Equipamiento que se relacionan directamente con el Puerto Terrestre de Los Andes, en el segundo caso los proyectos buscan resolver la conexión por una parte entre la Autopista troncal sur y la ruta CH-60, como también entre las comunas de Valparaíso y Viña del Mar.

La creciente cantidad de proyectos de gran envergadura dentro del área de influencia pueden ser entendidos como parte de una estrategia para insertarse en las redes económicas de la globalización asociadas al IIRSA.

El análisis permite verificar que existe una relación espacial directa entre las redes trazadas por la iniciativa de infraestructura y los proyectos adyacentes a las redes, siendo necesario analizar el tipo de territorio que afecta a nivel local, considerando la gran biodiversidad que posee la Región de Valparaíso y sus características naturales. Para ello, en la Figura 3 se observa el área de influencia de 10 kilómetros a partir de los proyectos red de la IIRSA en la Región y cómo estos se sobreponen o no con las áreas protegidas más cercanas a la red.

Comenzando por la ruta de San Antonio-San Fernando, localizada al Suroeste de la Región, se puede observar que a 10 kilómetros de distancia se encuentra el Reserva Nacional y Sitio Ramsar El Yali, interviniendo $6,1 \mathrm{~km}^{2}$ de un área protegida que contiene uno de los 56 sitios de la Estrategia Regional para la Conservación de la Biodiversidad.

Por otra parte, la ruta $\mathrm{CH}-60$ que atraviesa la Región de Valparaíso desde los Andes hasta la costa interviene directamente o mediante el área de influencia 6 Áreas Protegidas. Cercano al proyecto IIRSA para la optimización del Sistema Cristo Redentor en la provincia de Los Andes, se encuentra el Sitio Ramsar Juncal, con un área de $148,243 \mathrm{~km}^{2}$, de estos, el $62 \%$ se encuentra intervenido. En la misma provincia se encuentra la Reserva Natural Río Blanco con una extensión de $44,9 \mathrm{~km}^{2}$, superficie total que se encuentra dentro del área de influencia adyacente a la ruta IIRSA. El Sitio Prioritario Cordillera El Melón cuenta con una superficie intervenida de $109,5 \mathrm{~km}^{2}$ correspondientes al $17 \%$ de su territorio total. El área protegida más grande corresponde a la Reserva de la Biosfera Campana-Peñuelas, incluyendo como su nombre lo menciona por un sector el Parque Nacional La Campana y, por otro, la Reserva Natural Lago Peñuelas, en el primer caso la superficie intervenida es el $10 \%$ de una de las Zonas Núcleos de la Reserva, mientras que en la segunda el área comprometida equivale al 8\% del total, no obstante este tipo de protección incluye Zonas de Amortiguación y Transición sumando $2.368 \mathrm{~km}^{2}$, dentro de estos el $27 \%$ se ve afectado por la fragmentación que provoca la ruta $\mathrm{CH}-60$ en el sector La Campana. Finalmente, en la comuna de Valparaíso se ubica el Sitio Prioritario Laguna Verde, donde el $4 \%$ se encuentra dentro del área de influencia, cercano a su vez de los proyectos IIRSA que buscan la ampliación del Puerto de Valparaíso.

De esta forma se puede observar cómo la ejecución de proyectos globales intervienen de diversas maneras los territorios locales, ya sea aumentando el valor de sus suelos para el desarrollo industrial o afectando a aquellos en que precisamente su valor no es monetario, sino que ecológico, del que la preservación es un punto de intransigencia por parte 


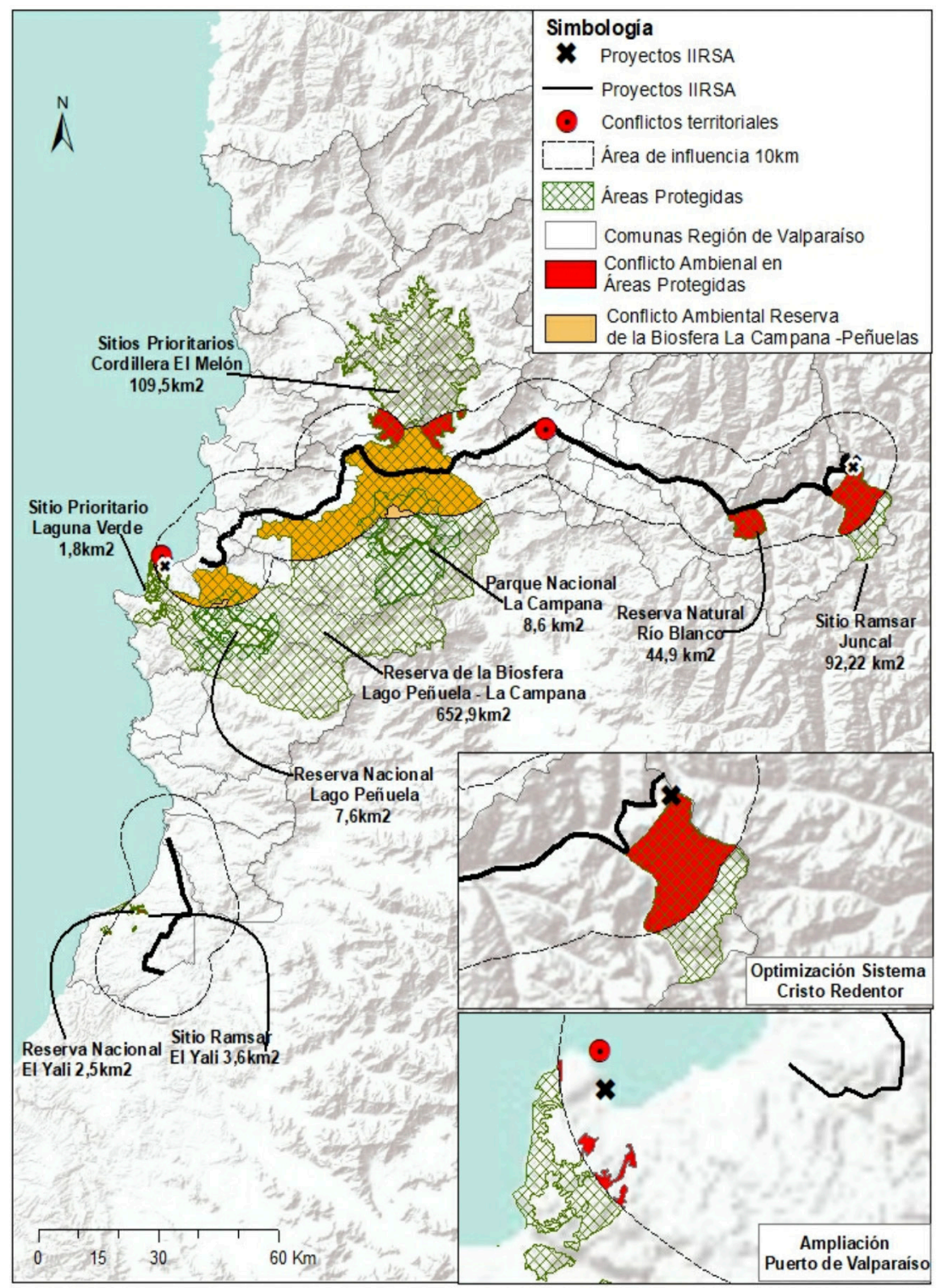

Figura 3. Conflictos ambientales y territoriales en relación con el IIRSA en la Región de Valparaíso. Fuente: Elaboración propia.

de muchos, generando conflictos ambientales. Por otra parte, se puede observar en la Figura 2, dos sectores en conflicto, ligados directamente con la estrategia IIRSA, ambos proyectos corresponden al sector transporte y se encuentran concesionados al grupo OHL.

El primer caso se trata sobre la ampliación del Puerto de Valparaíso, parte importante según el Estado por movilizar por medio del transporte marítimo gran parte del comercio nacional e internacional, sin embargo, la realidad que se refleja en la ciudad corresponde a la gran pérdida de territorio costero, por concesiones privadas con representación del Estado que promueven megaproyectos, desencadenando tensiones en la sociedad a partir de la discusión de aquello que es público-privado en la ciudad. Mientras que, en el segundo caso, se trata de un sector de la ruta internacional $60-\mathrm{CH}$, específicamente entre el tramo San Felipe-Panquehue, la importancia del 
conflicto recae en cómo interviene un proyecto global en lo local, afectando a un grupo de actores y desencadenando movilizaciones sociales acerca de aquello que es mejor para la población.

Dentro de los proyectos en la región, existen 2 iniciativas que consideramos emblemáticas, tanto por sus montos de inversión como por sus impactos territoriales en la dinámica de la región; a) el corredor bioceánico y b) la ampliación del puerto de Valparaíso.

\section{Corredor Bioceánico}

El proyecto del corredor bioceánico concentra sus esfuerzos en el paso de la cordillera de los Andes por un ferrocarril de alta velocidad, que permite transportar camiones de carga junto con sus containers. Se pretende así superar las dificultades climáticas que mantienen cerrada durante la época de invierno esta sección de la cordillera debido a que los caminos se encuentran cubiertos de nieve, generando pérdidas millonarias para las empresas que utilizan dicho paso. Así como señalan los informes generados por el Estado:

\section{El Área Bilateral Cristo Redentor signifi- ca, sin desmedro de las restantes, la más importante vinculación histórica, cultural, económica y de infraestructura entre Chile y Argentina. Además de concentrar los movimientos mayoritarios de cargas y personas provenientes de la Argentina, Uruguay y Brasil, la relación entre Mendoza y Chile es, además de histórica, fructífera y frecuente. Probablemente constituya la imagen objetivo que pueda orientar una estrategia de conjunto (Ministerio de Obras Públicas, 2012).}

En efecto, esta sección del corredor bioceánico encuentra su antecedente en el ferrocarril transandino que fue construido a fines del siglo XIX y que se mantuvo activo hasta la segunda mitad del siglo $\mathrm{XX}$. Dicho proyecto fue construido por la Empresa Argentina Hermanos Clark, con fondos europeos, estatales, y otros financiamientos privados. Este proyecto fue contemplado como una de las obras públicas más relevantes de su época.

La iniciativa, que surge de la Corporación América, fue aprobada por los gobiernos de Chile y Argentina en el 2008. En el 2009 se firma un acuerdo de cooperación entre ambos países en el mismo lugar donde se abrazó O'Higgins y San Martín. Con esto se crea una entidad binacional con capacidad de gestionar y controlar los avances. También se ampara en el tratado internacional aprobado por las legislaturas de Argentina y Chile.

La Corporación América creó un consorcio internacional a las que sumó la Empresas Navieras S.A. (Chile), Mitsubishi Corporation (Japón), GEODATA (Italia), Contreras Hermanos (Argentina).

Este, al igual que otros proyectos, plantea la necesidad de superar las limitaciones geográficas para el desarrollo económico, de forma particular la cordillera de los Andes, sus condiciones físicas y climáticas se presentan como barreras para la movilidad de mercancías. De esta forma, el desarrollo de las técnicas asociadas al transporte busca superar dichas barreras.

Al mismo tiempo, recrean un imaginario territorial de grandes áreas donde existen vacíos demográficos (Porto-Gonçalves y Quental, 2012), y particularmente para el eje Mercosur-Chile promueven la superación de las limitaciones que impone la naturaleza. Estas estrategias discursivas tienen como objetivo esconder las consecuencias respecto de áreas ricas en biodiversidad y poblaciones. Precisamente los proyectos se centran en dar cuenta de la logística de las redes, no contemplando sus implicancias territoriales.

Detrás de este proyecto se busca un reposicionamiento de la geopolítica global en ámbito de grandes potencias del Asia-Pacífico, donde Brasil intenta instalar su potencial. Esta lógica no es nueva, sino por el contrario -como ha sido posible evidenciar el discurso geopolítico en Brasil, Argentina y Chile-, posee una historia común desde las dictaduras latinoamericanas donde existió una colaboración conjunta para instalar la violencia política.

Lo que se busca es construir un corredor logístico para:

1. Unir centros de consumo masivos de mercaderías de alto valor agregado

2. Unir centros de producción, polos industriales y centros de consumo

3. Unir puertos de ambos océanos, pudiendo generar un flujo de ida y vuelta

4. Concepto de puertos secos como concentradores y reductores de costos logísticos para flujos de cargas para el comercio exterior 
La principal exportación de Brasil es petróleo y automóviles, y la de Argentina soja y sus derivados, principalmente con dirección a China. El 50\% de los intercambios de Argentina a Chile son hidrocarburos (Ministerio de Obras Públicas, 2012)

\section{Ampliación del Puerto de Valparaíso}

Otro de los hitos relevantes dentro del eje Mercosur-Chile es la ampliación del puerto de Valparaíso, que ha tenido significativas críticas por parte de actores locales de carácter público y social que se oponen al desarrollo de este proyecto.

Cuando son definidas las oportunidades de la región de Valparaíso en los instrumentos de planificación, uno de los primeros puntos señalados es que la región cuenta con el principal complejo portuario del país y de la costa oeste sudamericana (Ministerio de Obras Públicas, 2009). Dicha oportunidad identificada por los planificadores nacionales se articula con los intereses ya mencionados de fortalecer el flujo de mercancías hacia el Pacífico sur.

El proyecto busca incrementar la capacidad de carga del puerto de Valparaíso, el que si bien está posicionado dentro de los puertos en el movimiento de contenedores de América del Sur, como lo muestra la Tabla 3, aún se encuentra distante de los principales puertos del eje MCC (Santos y Buenos Aires).

Lo que se pretende es consolidar un "Puerto de Gran Escala", que en esta etapa consiste en la modernización del terminal 2 del puerto, mediante la ampliación a 12,5 hectáreas de superficie del terminal (OHL, 2017). Con esto se conseguiría elevar la capacidad de transferencia de carga para 1.150.000 de TEU por año. De esta forma, sumado al proyecto "Puerto Central" de San Antonio, el complejo portuario sumará 2 millones de TEU/año a su capacidad actual (Michea, 2013). De concretarse ambos proyectos, casi se duplicaría la capacidad actual de movilización de contenedores entre ambos puertos, que al 2012 movilizaba 2.011.918 TEU anuales (Michea, 2013). Esto da cuenta de una alta expectativa de las autoridades nacionales y de la administración portuaria acerca del incremento de la demanda de exportaciones que son embarcadas desde la región de Valparaíso.

En la actualidad, este proyecto forma parte de una de las discusiones relevantes a nivel local, dividiendo de forma significativa a los habitantes frente a la aprobación y rechazo de la iniciativa, lo que ha generado una creciente discusión pertinente a la iniciativa (Texido, 2014; Alarcón, 2009). El debate gira en torno al impacto del proyecto en la ciudad de Valparaíso y sus alcances reales para generar un impulso social y económico en la comuna. En este escenario, llama la atención en esta discusión la ausencia de vinculación entre el objetivo de incrementar la capacidad portuaria y el rol que posee la estrategia IIRSA, que tan solo ha sido señalada desde algunos movimientos sociales locales, apareciendo de forma marginal una crítica al plan IIRSA y sus consecuencias en la región.

Es importante mencionar que la dimensión de este proyecto hace que sus impactos no se restrinjan al área urbana de la comuna de Valparaíso (como queda evidenciado en el mapa 2). Muestra de esto es la proyección de habilitar una cantera en el sector de Laguna Verde, para abastecer de áridos para la construcción del Terminal 2. No obstante, en la localidad hubo una severa oposición argumentando los graves impactos socioambientales de dicha cantera, lo que llevó a definir un nuevo lugar de extracción de materiales.

Tabla 3. Movimiento de contenedores en los principales puertos marítimos del Eje MERCOSUR-Chile (T.E.U.*).

\begin{tabular}{lrrr}
\hline Puerto & \multicolumn{1}{c}{2010} & \multicolumn{1}{c}{2011} & 2012 \\
\hline Santos & 2.715 .568 & 2.985 .922 & 2.961 .426 \\
Buenos Aires (incluye Exolgan) & 1.730 .831 & 1.851 .687 & 1.656 .428 \\
San Antonio & 870.719 & 928.432 & 1.069 .271 \\
Valparaíso & 878.787 & 973.012 & 860.623 \\
Montevideo & 671.952 & 861.164 & 753.889 \\
Rio Grande & 647.188 & 618.039 & 611.133 \\
Itajaí & 38.495 & 438.752 & 385.193 \\
Zárate & 86.814 & 107.928 & 123.974 \\
Paranaguá & 546.564 & 681.678 & 74.383 \\
\hline
\end{tabular}

* Twenty-foot equivalent unit (TEU). Capacidad de carga de un contenedor normalizado de 20 pies de largo.

Fuente: Valdés y Parimbelli, 2014. 


\section{Conclusiones}

Los resultados de este estudio permiten evidenciar el modo en que los discursos de desarrollo geopolítico promueven al eje Mercosur-Chile como una de las piezas fundamentales para la inserción de América Latina en las economías mundo de la cuenca del pacifico.

Al mismo tiempo, dan cuenta de las representaciones hegemónicas de carácter político y económico, cuyo discurso geopolítico se sostiene a través el trinomio conceptual: desarrollo, integración y territorio. Los cuales son entendidos respectivamente como sinónimos de: crecimiento económico, conectividad y superación barreras naturales para la producción económica.

Esta perspectiva economicista refuerza la dicotomía tradicional de la modernidad que existe entre cultura y naturaleza, promoviendo el dominio y control de la naturaleza. De esta forma, se identifica especialmente la comprensión de la cordillera de Los Andes en cuanto barrera natural para el desarrollo, y al mismo tiempo, el borde costero en cuanto oportunidad para la integración con la cuenca del Pacifico. Es así como surgen los proyectos asociados al paso Cristo Redentor que promueven la superación de las debilidades logísticas, generadas por las restricciones al transporte que genera la cordillera de los Andes. Así como también, la expansión del puerto de Valparaíso, como forma de potenciar las fortalezas logísticas que posee la región.

Sin embargo, los resultados del análisis empírico permiten evidenciar la incongruencia entre los discursos de desarrollo y los impactos reales que estos proyectos generan en términos socio-ambientales. En efecto, los efectos generados por el eje sobre las áreas de conservación ambiental son significativos, y hasta el momento han sido escasamente discutidos por actores institucionales y sociales presentes en estos territorios. Al mismo tiempo, el desajuste entre discurso e intervención, se evidencia en las reestructuraciones territoriales generadas a escala local, como ocurre en la ciudad de Valparaíso ante la expansión de la puerto de Valparaíso, generando discrepancias entre los actores sociales que aprueban, y los que se oponen al de proyectos, exponiendo problemas de gobernanza, en que los capitales con los que cuentan los promotores de las inversiones resultan decisivos en la instauración de los modelos de desarrollo local. Al mismo tiempo, la falta de conocimiento e información de los actores locales frente a la IIRSA plantean desequilibrios en el capital cultural con el que cuentan estos actores.

A partir de esto se vuelve indispensable avanzar en una lectura transescalar de los impactos generados por las redes del IIRSA, analizando los proyectos no de forma aislada y fragmentada, sino que en relación con el resto de las iniciativas que están en curso.

\section{Referencias Citadas}

Aguiar, Diana

2017 A geopolítica de infraestrutura da China na América do Sul: um estudo a partir do caso do Tapajós na Amazônia brasileira. Rio de Janeiro, Brasil, FASE/ActionAid.

Alimonda, Héctor

2011 "La colonialidad de la naturaleza. Una aproximación a la ecología política latinoamericana". Pp. 21-58 En: La naturaleza colonizada. Ecología Política y Minería en América Latina. Buenos Aires: Ediciones CICCUS y CLACSO. BNDES

2003 I Seminário Internacional de Co-financiamento BNDES/ CAF: Prospecção de Projetos de Integração Fisica SulAmericana. Íntegra do Discurso do presidente do BNDES, Carlos Lessa. Retrieved July 29, 2017: http://www.bndes. gov.br/SiteBNDES/export/sites/default/bndes_pt/Galerias/ Arquivos/conhecimento/seminario/caf_abertura1.pdf

Camacho, GH and S. Molina

2005 IIRSA y La Integración Regional. OSAL. Retrieved June 14, 2017 (http://biblioteca.clacso.edu.ar/gsdl/collect/ clacso/index/assoc/D3842.dir/41Camach.pdf).
Ceceña, A.E.; P. Aguilar Y C. Motto

2007 Territorialidad de la dominación. Integración de la Infraestructura Regional Sudamericana (IIRSA). Buenos Aires: Observatorio Latinoamericano.

Couto, L.F.

2008 A Iniciativa Para a Integração Da Infra-Estrutura Regional Sul-americana-IIRSA Como Instrumento Da Política Exterior Do Brasil Para a América Do Sul. Rio de Janeiro: OIKOS http://www.revistaoikos.org/seer/index. php/oikos/article/viewArticle/109.

Dijck, P. Van

2008 "Troublesome Construction: The Rationale and Risks of IIRSA". European Review of Latin American And. Retrieved June 14, 2017 (http://erlacs.ubiquitypress.com/ articles/10.18352/erlacs.9621/galley/10040/download/).

Dijck, van

2013 The Impact of the IIRSA Road Infrastructure Programme on Amazonia. Routledge. Retrieved April 24, 2018 (https:// books.google.cl/books?id=_nJ_F-0u4OAC\&printsec=fron tcover $\& \mathrm{hl}=\mathrm{es} \# \mathrm{v}=$ onepage $\& \mathrm{q} \& \mathrm{f}=$ false) . 
GORE-Gobierno Regional de Valparaíso

2012 Estrategia Regional de Desarrollo 2020. Recuperado de http://www.subdere.gov.cl/sites/default/files/documentos/ estrategia_regional_de_desarrollo_region_de_valparaiso. pdf

Escobar, A.

2007 La Invención Del Tercer Mundo: Construcción y Deconstrucción Del Desarrollo. Caracas: Fundación Ed. el Perro y la Rana. Retrieved June 8, 2017 (https://books. google.cl/books?hl=es\&lr=\&id=P_aapX4T8qEC\&oi=fnd \&pg=PA7\&ots=hoje102VtC\&sig=tz1PFUwRm99Z0hmh MIEi9UBgqPk).

Gallardo, R.; Vyoleta, M.; Peña González, R. y González Ulloa

Aguirre, P.A.

2012 "Brasil: raíces geopolíticas y actual influencia en expansión". Política y cultura (37), 233-253.

Harvey, D.

2005 O novo imperialismo. São Paulo: Ediciones Loyola.

Madrid, Claudia Kis; Hickey, Gordon; Bouchard, Michel A.

2011 "Strategic Environmental Assessment Effectiveness and the Initiative for the Integration of Regional Infrastructure in South America (Iirsa): A Multiple Case Review”. J. Env. Assmt. Pol. Mgmt. 13(515). Retrieved June 14, 2017.

Marini, Ruy Mauro

1977 "La acumulación capitalista mundial y el subimperialismo". Cuadernos Políticos, (12), México.

Martínez, G. y J. Houghton

2008 "La IIRSA: O El Mega-Ordenamiento de Los Territorios Indígenas". De Los Pueblos. Retrieved June 14, 2017 (http://observatorioetnicocecoin.org.co/cecoin/ files/Tierra_contra_la_muerte.pdf\#page=231).

Menna, $\mathrm{M}$.

2008 "Sobre La Nueva Conquista Del Desierto: El IIRSA En La Patagonia Argentina". Revista Theomai. Retrieved June 14, 2017 (http://revista-theomai.unq.edu.ar/NUMERO18/ Menna.pdf).

Michea, A.

2013 Puerto de Gran Escala: The case for a New Container Terminal in Central Chile. International Transport Forum Discussion Paper, OECD. DOI:http://dx.doi. org/10.1787/5jz40rzftr0x-en

Ministerio de Obras Públicas \& Ministerio de Planificación

Federal, Inversión Pública y Servicios

2012 Estudio Binacional de Conectividad Argentina-Chile: ATN/OC10847RG Servicios de Consultoría.

Ministerio de Obras Públicas

2009 Actualización Plan Director de Infraestructura: Informe Final Metodología General del PDI.

Montañez, G., y Delgado, O.

1998 "Espacio, territorio y región: conceptos básicos para un proyecto nacional". Cuadernos de Geografía, VII (1-2),120-134.

Moreira, MM.

2007 Trade Costs and the Economic Fundamentals of the Initiative for Integration of Regional Infrastructure in South America (IIRSA)(Working Paper ITD=. Retrieved June 14, 2017 (https://books.google.cl/books?hl=es\&lr=\&id=IIqt4Ne1QHkC\&oi=fnd\&pg=PA2\&dq=+iirsa\&ots=t3QceIC qmb\&sig=cRXNt-ZP7ptTEX7rtIchH3s1uwU).

Moreno, $\mathrm{C}$.

2015 O Brasil made in China: para pensar as reconfigurações do capitalismo contemporâneo. Sao Paulo, Brasil: Fundação Rosa Luxemburg.
OHL

Sitio web institucional. Consultado el 13 de marzo de 2017.

Panez, A.; Mansilla, P. y Moreira, A.

2018 "Agua, tierra y fractura sociometabólica del agronegocio. Actividad frutícola en Petorca, Chile". Bitácora Urbano Territorial 28(3), 153-160.

Pieck, Sonja K.

2011 Beyond Postdevelopment: Civic Responses to Regional Integration in the Amazon. Journal of Cultural Geography 28(1):179-202. Retrieved April 24, 2018 (http:// www.tandfonline.com/doi/abs/10.1080/08873631.2011. 54849).

Porto-Gonçalves, C.

2006 A globalização da natureza e a natureza da globalização. Editora Civilização Brasileira. Rio de Janeiro.

Porto-Gonçalves, C. y de Araújo Quental, P.

2012 "Colonialidade do poder e os desafios da integração regional na América Latina". Polis. Revista Latinoamericana, 11 (31), 259-332.

Porto-Gonçalves, C.

2011 "Ou inventamos ou erramos-encruzilhadas da integração regional sul-americana”. En Rego, A., Silva, P. \& Bojikian, A. (Org.). Governança Global e Integração da América do Sul (pp. 133-176). Brasília: IPEA.

Quintanar, S. y López, R.

2003 "O Plano de Ação para a Integração da Infra-estrutura Regional Sul americana (IIRSA): oportunidades e riscos. Seu significado para o Brasil e a Argentina". Revista Brasileira de Política Internacional, 46(1), 213-221.

Quintero-Weir, José

2015 El Camino de Las Comunidades. 2nd ed. Cochabamba: Seminario de Integración Abya Yala Desde Abajo.

Rodríguez, Mabel Alarcón

2009 "Renovación Del Frente Portuario de Valparaíso. Oportunidad de Re-Articulación Puerto-Ciudad". Cuaderno Urbano 8(8):205-28. Retrieved June 13, 2018 (http://www.scielo.org.ar/scielo.php?pid=S1853$36552009000100010 \&$ script=sci_arttext\&tlng=en).

Svampa, M.

2008 "La Disputa Por El Desarrollo: Territorio, Movimientos de Carácter Socio-Ambiental y Discursos Dominantes". Escuela, Conocimiento Público y Disputas de Poder. Retrieved June 14, 2017 (http://www.cronicon.net/paginas/ Documentos/paq2/No.24.pdf).

Svampa, M. y Slipak, A.

2015 "China en América Latina: Del Consenso de los Commodities al Consenso de Beijing". Revista Ensambles, (3), 34-63.

Silveira, ML.

2007 Los Territorios Corporativos de La Globalización. Geograficando. Retrieved July 4, 2017 (http://www.memoria. fahce.unlp.edu.ar/library? $\mathrm{a}=\mathrm{d} \& \mathrm{c}=\operatorname{arti} \& \mathrm{~d}=\mathrm{Jp}$ r3665).

Silveira, ML.

2008 "Globalización y Territorio Usado: Imperativos y Solidaridades". Cuadernos Del CENDES. Retrieved July 4, 2017 (http://www.redalyc.org/html/403/40311392002/).

Tautz, C

2009 Da Alca à IIRSA. Le Monde Diplomatique Brasil. Retrieved June 14, 2017 (http://www.autresbresils.net/IMG/ pdf/alcairsaa.pdf). 
Texido, Alberto and Paola de la Sotta

2014 "Las barreras institucionales que dificultan la inclusión de Valparaíso Ciudad y Puerto". Revista Márgenes Espacio Arte y Sociedad 11(15):70-74. Retrieved June 13, 2018 (http://200.14.68.74/index.php/margenes/article/view/305).

Valdés, J. y Parimbelli, M.

2014 Caracterización Socioeconómica y Ambiental Eje Mercosur-Chile. Foro Técnico IIRSA, UNASUR-Cosiplan.

Van Dijck, $P$

2013 The impact of the IIRSA road infrastructure programme on Amazonia. Routledge. Retrieved from https://books. google.cl/books?id=_nJ_F-0u4OAC\&printsec=frontcove $\mathrm{r} \& \mathrm{hl}=\mathrm{es} \# \mathrm{v}=$ onepage $\& \mathrm{q} \& \mathrm{f}=$ false
Wallerstein, I.

2007 "La situación mundial frente al declive de Estados Unidos”. En: Gandásegui, M. (Coord.) Crisis de hegemonía de Estados Unidos (pp. 95-102). México, Siglo XXI \& CLACSO.

\section{Zibechi, R.}

2006 "IIRSA: La Integración a La Medida de Los Mercados". Ecología Política. Retrieved June 14, 2017 (https://dialnet. unirioja.es/descarga/articulo/2259975.pdf).

Zibechi, R.

2012 Brasil Potencia. Entre la integración regional y un nuevo imperialismo. Santiago de Chile: Editorial Quimantu.

\section{Notas}

1 Entendemos el concepto de "acumulación por desposesión" a partir del trabajo de David Harvey (2005), quien hace una relectura de la teoría general de la acumulación de capital de Marx, el que hace la distinción entre acumulación originaria o primitiva y acumulación como reproducción ampliada. Harvey señala que en las últimas décadas una de las estrategias predominantes para incrementar la acumulación implica una desposesión en la medida en que el excedente de capital dinero que carece de oportunidades de inversión productiva y rentable en los países capitalistas centrales, presiona mediante el pillaje y la violencia para insertarse en nuevas latitudes o ámbitos de la vida social (educación, agua potable, previsión social, etc.).

2 Acerca del tema del apoyo de Estados Unidos al golpe de Estado de 1964, se recomienda revisar el riguroso trabajo plasmado en el documental "O dia que durou 21 anos" ("El día que duró 21 años”, 2012) dirigido por Camilo Tavares.
3 Para implementar dicho proceso de modernización el régimen llevó a cabo un proceso sistemático de represión, bajo la doctrina de Seguridad Nacional, para contener a las fuerzas opositoras a esta "alianza nacional".

4 Un dato ilustrativo de este intento de consolidación económica, es que en 2011 las multinacionales brasileñas repatriaron a Brasil 21.200 millones de dólares, cifra que equivale al PIB anual de Paraguay. Una parte sustancial fue retornada desde los países latinoamericanos, donde las multinacionales brasileñas tienen sus mayores inversiones (Zibechi, 2012: 14).

5 Que pueden ser identificadas claramente en el Libro Blanco sobre América Latina y el Caribe escrito por China.

6 Información online obtenida a partir del Sistema de Información de Proyectos, según el Consejo Suramericano de Infraestructura y Planeamiento (COSIPLAN). 
